\title{
NULL CONTROLLABILITY OF LINEAR HEAT AND WAVE EQUATIONS WITH NONLOCAL SPATIAL TERMS*
}

\author{
ENRIQUE FERNÁNDEZ-CARA ${ }^{\dagger}$, QI LÜ๋, AND ENRIQUE ZUAZUA ${ }^{\S}$
}

\begin{abstract}
In this paper, we study the null controllability of linear heat and wave equations with spatial nonlocal integral terms. Under some analyticity assumptions on the corresponding kernel, we show that the equations are controllable. We employ compactness-uniqueness arguments in a suitable functional setting, an argument that is harder to apply for heat equations because of its very strong time irreversibility. Some possible extensions and open problems concerning other nonlocal systems are presented.
\end{abstract}

Key words. null controllability, heat equation, wave equation, nonlocal terms

AMS subject classifications. 93B05, 93B07

DOI. $10.1137 / 15 \mathrm{M} 1044291$

1. Introduction and statement of the problems. Let $\Omega \subset \mathbf{R}^{N}$ be a bounded domain $\left(N \geq 1\right.$ is an integer), with boundary $\partial \Omega$ of class $C^{2}$. We fix $T>0$ and set $Q:=\Omega \times(0, T)$ and $\Sigma:=\Gamma \times(0, T)$. We also consider a nonempty (small) open set $\omega \subset \Omega$. As usual, $1_{\omega}$ denotes the characteristic function of $\omega$.

Let $K=K(x, \xi)$ belong to $L^{2}(\Omega \times \Omega)$. In this paper, we will consider the following controlled heat and wave equations involving nonlocal in space terms:

$$
\begin{cases}y_{t}-\Delta y+\int_{\Omega} K(x, \xi) y(\xi, t) d \xi=v 1_{\omega} & \text { in } Q, \\ y=0 & \text { on } \Sigma, \\ y(x, 0)=y^{0}(x) & \text { in } \Omega\end{cases}
$$

and

$$
\begin{cases}y_{t t}-\Delta y+\int_{\Omega} K(x, \xi) y(\xi, t) d \xi=v 1_{\omega} & \text { in } Q, \\ y=0 & \text { on } \Sigma, \\ y(x, 0)=z^{0}(x), \quad y_{t}(x, 0)=z^{1}(x) & \text { in } \Omega .\end{cases}
$$

In (1) (resp. (2)), $v$ is a distributed control acting only on the set $\omega \times(0, T)$, and $y$ (resp. $\left.\left(y, y_{t}\right)\right)$ is the state.

\footnotetext{
${ }^{*}$ Received by the editors October 19, 2015; accepted for publication (in revised form) April 8, 2016; published electronically August 9, 2016.

http://www.siam.org/journals/sicon/54-4/M104429.html

${ }^{\dagger}$ Departamento EDAN and IMUS, Universidad de Sevilla, Aptdo. 1160, 41080 Sevilla, Spain (cara@us.es). This author was partially supported by MINECO (Spain) grant MTM2013-41286-P.

${ }^{\ddagger}$ School of Mathematics, Sichuan University, Chengdu, 610064, China (lu@scu.edu.cn). This author was supported by the NSF of China under grant 11471231, the Fundamental Research Funds for the Central Universities in China under grant 2015SCU04A02, and MICINN (Spain) grant MTM2011-29306-C02-00.

§Departamento de Matemáticas, Universidad Autónoma de Madrid, Cantoblanco, 28049 Madrid, Spain (enrique.zuazua@uam.es). This author was partially supported by the advanced grant NUMERIWAVES/FP7-246775 of the European Research Council Executive Agency, FA9550-14-10214 of EOARD-AFOSR, FA9550-15-1-002 of AFOSR, MTM2011-29306 and MTM2014-52347 grants of MINECO, and a Humboldt Award at the University of Erlangen-Nürnberg. This work was done while the author was visiting Sichuan University, Chengdu, China.
} 
If $y^{0} \in L^{2}(\Omega)$ and $v \in L^{2}(\omega \times(0, T))$, then (1) possesses a unique weak solution $y$, with $y \in L^{2}\left(0, T ; H_{0}^{1}(\Omega)\right) \cap C^{0}\left([0, T] ; L^{2}(\Omega)\right)$ and satisfies classical "energy" estimates; actually, this remains true if $v 1_{\omega}$ is replaced by a general right-handside $f \in L^{2}\left(0, T ; H^{-1}(\Omega)\right)$.

On the other hand, if $\left(z^{0}, z^{1}\right) \in H_{0}^{1}(\Omega) \times L^{2}(\Omega)$ and $v \in L^{2}(\omega \times(0, T))$, (2) also possesses a unique weak solution $y$, with $\left(y, y_{t}\right) \in C^{0}\left([0, T] ; H_{0}^{1}(\Omega) \times L^{2}(\Omega)\right)$.

This paper is mainly devoted to the analysis of the controllability properties of (1) and (2).

Definition 1. It will be said that (1) is null-controllable at time $T$ if for any $y^{0} \in L^{2}(\Omega)$, there exist controls $v \in L^{2}(\omega \times(0, T))$ such that the associated states satisfy

$$
y(x, T)=0 \quad \text { in } \Omega .
$$

Similarly, (2) is null-controllable at time $T$ if for any $\left(z^{0}, z^{1}\right) \in H_{0}^{1}(\Omega) \times L^{2}(\Omega)$, there exist controls $v \in L^{2}(\omega \times(0, T))$ such that the associated states satisfy

$$
y(x, T)=0, \quad y_{t}(x, T)=0 \quad \text { in } \quad \Omega .
$$

Since (2) is linear and reversible in time, it is null-controllable if and only if it is exactly controllable, i.e. if and only if, for any $\left(z^{0}, z^{1}\right)$ and $\left(w^{0}, w^{1}\right)$ in $H_{0}^{1}(\Omega) \times L^{2}(\Omega)$, there always exist controls $v \in L^{2}(\omega \times(0, T))$ such that the associated solutions satisfy

$$
y(x, T)=w^{0}(x), \quad y_{t}(x, T)=w^{1}(x) \text { in } \Omega .
$$

The study of the controllabilty of (1) and (2) is motivated by many relevant applications from physics and biology; see, for instance, [8, 9]. However, to our knowledge, very few results are available. As far as we know, [4] is the unique published work on this subject; it concerns the exact controllability of the Schrödinger equation with a nonlocal term.

Before presenting the main results of this paper, we introduce some notation and the main conditions that the kernel $K$ will be required to fulfill.

Throughout the paper, $C$ denotes a generic positive constant depending on $\Omega, \omega$ and maybe other data, which may vary from line to line (unless otherwise stated). Sometimes, we will emphasize the fact that $C$ depends on (say) $T$ by writing $C(T)$. For all $m \geq 1$, the inner product and norm in $L^{2}(\Omega)^{m}$ will be respectively denoted by $(\cdot, \cdot)$ and $\|\cdot\|$; on the other hand, $|\cdot|$ will stand for the Euclidean norm in $\mathbf{R}^{m}$.

We will denote by $\lambda_{1}, \lambda_{2}, \ldots$ (resp. $\phi_{1}, \phi_{2}, \ldots$ ) the eigenvalues (resp. the unit $L^{2}$ norm eigenfunctions) of the Dirichlet Laplacian in $\Omega$. Recall that $0<\lambda_{1}<\lambda_{2} \leq$ $\lambda_{3} \leq \cdots, \lambda_{m} \sim m^{2 / N}$ as $m \rightarrow+\infty$ and $\phi_{1}>0$ in $\Omega$.

We impose the following conditions on the kernel $K$ :

$$
K \in L^{2}(\Omega \times \Omega), \quad x \mapsto \int_{\Omega} K(\xi, x) f(\xi) d \xi \text { is analytic for all } f \in L^{2}(\Omega) .
$$

This is an analyticity assumption in the second variable. It will play a key role when proving unique continuation properties for the adjoint system when deriving observability inequalities. Indeed, as in the context of linear PDEs, the problem of controllability under consideration is equivalent to a suitable observability inequality for the corresponding adjoint system. The main argument we shall employ to derive these observability inequalities will be of a perturbation nature, the so-called compactness-uniqueness argument, that will allow us to transfer known observability 
inequalities for the heat and wave equations into the corresponding nonlocal problems. For this argument to be applied one needs a unique continuation property to be proved. In the context of classical PDEs models this unique continuation is achieved by means of Holmgren's Uniqueness Theorem or Carleman inequalities. But these techniques do not apply directly for PDE involving nonlocal terms. This is why we require the strong analyticity condition (3).

In the context of the wave equation, this compactness-uniqueness argument can be applied in the energy space and the analyticity condition above, together with the classical geometric control condition $(G C C)$ ensuring the controllability of the wave equation, suffices for the result to hold for the nonlocal model.

The argument is more subtle for the heat equation. Indeed, in this case, due to the very strong time irreversibility, the observability inequality holds only in a very weak space involving a weighted norm of the initial data with weights that decay exponentially at high frequencies. Accordingly, further technical assumptions on the kernel $K$ will be needed so that the perturbation argument can be applied in these weak spaces.

The rest of the paper is organized as follows. In section 2, we analyze in detail the heat equation, establish the corresponding controllability result, and give its proof, following the lines indicated above. Section 3 is devoted to the wave equation. Finally, some additional comments, open questions and further applications are indicated in section 4 .

2. The nonlocal heat equation. In order to indicate the assumptions that, together with (3), must be satisfied by the kernel $K$, we recall the following spectral observability inequality (see [6, Remark 6.1]), that plays a key role in the analysis of the null controllability of heat equations with time-independent coefficients that permit a Fourier series representation.

Lemma 2. There exist $R(\Omega, \omega, T), C(\Omega, \omega)>0$ such that, for all $f \in L^{2}(\Omega)$, one has

$$
\sum_{j \geq 1} e^{-2 R \sqrt{\lambda_{j}}}\left|\left(f, \phi_{j}\right)\right|^{2} \leq C \iint_{\omega \times(0, T)}\left|\sum_{j \geq 1}\left(f, \phi_{j}\right) e^{-\lambda_{j}(t-T)} \phi_{j}(x)\right|^{2} d x d t .
$$

Our first main result deals with the null controllability of the nonlocal system (1). It is the following.

Theorem 3. Assume that (3) holds. Furthermore, assume that

$$
\left\{\begin{array}{l}
K(x, \xi)=\sum_{m, j \geq 1} k_{m j} \phi_{m}(x) \phi_{j}(\xi) \text { in } L^{2}(\Omega \times \Omega) \text { with } \\
\|K\|_{R}^{2}:=\sum_{m \geq 1}\left(\sum_{j \geq 1} \lambda_{j}^{-1}\left|k_{m j}\right|^{2}\right) \lambda_{m}^{-1} e^{2 R \sqrt{\lambda_{m}}}<+\infty,
\end{array}\right.
$$

where $R$ is the constant in Lemma 2. Then the system (1) is null-controllable.

As already mentioned, for the proof, the null controllability of (1) is reformulated in terms of the observability of the adjoint system, which is given by

$$
\begin{cases}-\varphi_{t}-\Delta \varphi+\int_{\Omega} K(\xi, x) \varphi(\xi, t) d \xi=0 & \text { in } Q \\ \varphi=0 & \text { on } \Sigma, \\ \varphi(x, T)=\varphi^{T}(x) & \text { in } \Omega .\end{cases}
$$


In other words, the main task reduces to prove the estimate

$$
\|\varphi(\cdot, 0)\|^{2} \leq C \iint_{\omega \times(0, T)}|\varphi|^{2} d x d t \quad \forall \varphi^{T} \in L^{2}(\Omega)
$$

for the solutions of the adjoint system (6).

Of course, the main difficulty in establishing this property is the presence of the nonlocal term.

Remark 4. Recall that the classical way to establish an estimate of this kind is to start from a global Carleman inequality of the form

$$
\iint_{Q} \rho^{-2}|\varphi|^{2} d x d t \leq C \iint_{\omega \times(0, T)} \rho^{-2}|\varphi|^{2} d x d t,
$$

where $C$ is independent of $\varphi^{T}$ and $\rho=\rho(x, t)$ is an appropriate weight function that blows up as $t \rightarrow T$; see the related results in $[7,6]$. However, it is not clear whether an approach of this kind can work in this framework, due to the presence of the nonlocal term. The reason is that the classical Carleman estimates for parabolic PDEs gives

$$
\begin{aligned}
& \iint_{Q} \rho^{-2}|\varphi|^{2} d x d t \leq C(\varepsilon) \iint_{\omega \times(0, T)} \rho^{-2}|\varphi|^{2} d x d t \\
& \quad+\varepsilon \iint_{\Omega \times(0, T)} \rho^{-2}\left|\int_{\Omega} K(\xi, x) \varphi(\xi, t)\right|^{2} d x d t .
\end{aligned}
$$

Here, $\varepsilon>0$ can be chosen arbitrarily small, but the second term in the right-hand side of (9) cannot be absorbed by the left-hand side, due to its nonlocal nature, contrary to what happens for classical pointwise lower order perturbations.

Remark 5. Note that, under the assumption (5), the kernel $K=K(x, \xi)$ also satisfies the following analyticity property in the first variable:

$$
x \mapsto \int_{\Omega} K(x, \xi) f(\xi) d \xi \text { is analytic for all } f \in H_{0}^{1}(\Omega) .
$$

Indeed, let $f \in H_{0}^{1}(\Omega)$ be given and let us set $A_{m}(f):=\sum_{j \geq 1} k_{m j}\left(\phi_{j}, f\right)$. Then

$$
\int_{\Omega} K(x, \xi) f(\xi) d \xi=\sum_{m \geq 1} A_{m}(f) \phi_{m}(x) .
$$

For all $m \geq 1$ and any $x \in \Omega$, we have

$$
\begin{aligned}
\left|A_{m}(f) \phi_{m}(x)\right| & \leq\left(\sum_{j \geq 1}\left|k_{m j}\right|\left|\left(\phi_{j}, f\right)\right|\right)\left|\phi_{m}(x)\right| \\
& \leq\left(\sum_{j \geq 1} \lambda_{j}^{-1}\left|k_{m j}\right|^{2}\right)^{1 / 2}\left(\sum_{j \geq 1} \lambda_{j}\left|\left(\phi_{j}, f\right)\right|^{2}\right)^{1 / 2}\left\|\phi_{m}\right\|_{L^{\infty}(\Omega)} \\
& \leq C(N, \epsilon)\|f\|_{H_{0}^{1}(\Omega)}\left(\sum_{j \geq 1} \lambda_{j}^{-1}\left|k_{m j}\right|^{2}\right)^{1 / 2} \lambda_{m}^{N / 2+\epsilon}
\end{aligned}
$$

for any small $\epsilon>0$. In view of (5), the series in (11) converges uniformly in $\Omega$. Consequently, (10) holds. 
Let us prove the observability estimate (7).

For any $\varphi^{T} \in L^{2}(\Omega)$, denote by $\varphi$ the solution to (6) and write

$$
\varphi=p+\zeta,
$$

where $p$ is the unique solution to

$$
\begin{cases}-p_{t}-\Delta p=0 & \text { in } Q \\ p=0 & \text { on } \Sigma \\ p(x, T)=\varphi^{T}(x) & \text { in } \Omega\end{cases}
$$

Obviously, one has

$$
\begin{cases}-\zeta_{t}-\Delta \zeta+\int_{\Omega} K(\xi, x) \zeta(\xi, t) d \xi=-\int_{\Omega} K(\xi, x) p(\xi, t) d \xi & \text { in } Q \\ \zeta=0 & \text { on } \Sigma, \\ \zeta(x, T)=0 & \text { in } \Omega .\end{cases}
$$

It is well known that the mapping

$$
\varphi^{T} \mapsto\left\|\varphi^{T}\right\|_{h}:=\left(\iint_{\omega \times(0, T)}|p|^{2} d x d t\right)^{1 / 2}
$$

is a norm in $L^{2}(\Omega)$ and, also, that there exist constants $C_{0}(\Omega, \omega, T)$ and $C_{1}(\Omega, \omega)$ such that

$$
\iint_{Q} e^{-2 C_{0} /(T-t)}|p|^{2} d x d t \leq C_{1}\left\|\varphi^{T}\right\|_{h}^{2} \quad \forall \varphi^{T} \in L^{2}(\Omega) .
$$

This is an immediate consequence of the global Carleman estimate that can be deduced for the solution to (12) (see [7] for details). Taking into account that

$$
p(x, t)=\sum_{j \geq 1} e^{-\lambda_{j}(T-t)}\left(\varphi^{T}, \phi_{j}\right) \phi_{j}(x) \text { in } L^{2}(Q),
$$

we see from Lemma 2 that

$$
\sum_{j \geq 1} e^{-2 R \sqrt{\lambda_{j}}}\left|\left(\varphi^{T}, \phi_{j}\right)\right|^{2} \leq C\left\|\varphi^{T}\right\|_{h}^{2} \quad \forall \varphi^{T} \in L^{2}(\Omega) .
$$

The inequality (7) will be a consequence of the following two estimates:

$$
\left\|\varphi^{T}\right\|_{h}^{2} \leq C \iint_{\omega \times(0, T)}|\varphi|^{2} d x d t \quad \forall \varphi^{T} \in L^{2}(\Omega)
$$

and

$$
\|\varphi(\cdot, 0)\|^{2} \leq C\left\|\varphi^{T}\right\|_{h}^{2} \quad \forall \varphi^{T} \in L^{2}(\Omega) .
$$

Let us give their proofs.

Proof of (16). Here, we argue by contradiction and we employ a compactnessuniqueness method.

Copyright $@$ by SIAM. Unauthorized reproduction of this article is prohibited. 
Let us assume that (16) does not hold. Then, for every $n \in \mathbf{N}$, we can find functions $\varphi_{n}^{T} \in L^{2}(\Omega)$ such that

$$
1=\left\|\varphi_{n}^{T}\right\|_{h}^{2}>n \iint_{\omega \times(0, T)}\left|\varphi_{n}\right|^{2} d x d t
$$

where $\varphi_{n}$ is the solution to (6) associated to $\varphi_{n}^{T}$.

Denote by $p_{n}\left(\right.$ resp. $\left.\zeta_{n}\right)$ the solution to (12) corresponding to $\varphi^{T}=\varphi_{n}^{T}$ (resp. the solution to (13) for $p=p_{n}$ ). Let us see that, at least for a subsequence, one must have

$$
\zeta_{n} \rightarrow 0 \text { strongly in } L^{2}(Q) \text {. }
$$

This will lead to a contradiction, since we will then have

$$
1=\left\|\varphi_{n}^{T}\right\|_{h}^{2}=\iint_{\omega \times(0, T)}\left|p_{n}\right|^{2} d x d t \leq 2 \iint_{\omega \times(0, T)}\left|\varphi_{n}\right|^{2} d x d t+2 \iint_{\omega \times(0, T)}\left|\zeta_{n}\right|^{2} d x d t
$$

and both terms in the right-hand side go to zero.

In order to prove (19), let us first check that

$$
\left\|\int_{\Omega} K(\xi, \cdot) p_{n}(\xi, \cdot) d \xi\right\|_{L^{2}\left(0, T ; H^{-1}(\Omega)\right)} \leq C \quad \forall n \in \mathbf{N} .
$$

This is implied by (15) and the assumption (5) on $K$ as follows:

$$
\begin{aligned}
\| \int_{\Omega} & K(\xi, \cdot) p_{n}(\xi, \cdot) d \xi \|_{L^{2}\left(0, T ; H^{-1}(\Omega)\right)}^{2} \\
& =\int_{0}^{T}\left(\sum_{\ell \geq 1} \lambda_{\ell}^{-1}\left|\left(\int_{\Omega} K(\xi, \cdot) p_{n}(\xi, t) d \xi, \phi_{\ell}\right)\right|^{2}\right) d t \\
& =\int_{0}^{T}\left(\sum_{\ell \geq 1} \lambda_{\ell}^{-1}\left|\sum_{m \geq 1} k_{m, \ell} e^{-\lambda_{m}(T-t)}\left(\varphi_{n}^{T}, \phi_{m}\right)\right|^{2}\right) d t \\
& \leq \sum_{\ell \geq 1} \lambda_{\ell}^{-1}\left(\sum_{m \geq 1}\left|k_{m, \ell}\right|^{2} \int_{0}^{T} e^{2 R \sqrt{\lambda_{m}}-2 \lambda_{m}(T-t)} d t\right)\left(\sum_{m \geq 1}\left|\left(\varphi_{n}^{T}, \phi_{m}\right)\right|^{2} e^{-2 R \sqrt{\lambda_{m}}}\right) \\
& \leq \frac{C_{1}}{2} \sum_{\ell \geq 1} \lambda_{\ell}^{-1}\left(\sum_{m \geq 1}\left|k_{m, \ell}\right|^{2} e^{2 R \sqrt{\lambda_{m}}} \lambda_{m}^{-1}\right)\left\|\varphi_{n}^{T}\right\|_{h}^{2} \\
& =\frac{C_{1}}{2}\|K\|_{R}^{2} .
\end{aligned}
$$

Hence, it can be assumed that $\zeta_{n}$ converges strongly in $L^{2}(Q)$.

Now, let us see that, at least for a subsequence, one has

$$
\varphi_{n} \rightarrow 0 \text { weakly in } L^{2}(\Omega \times(0, T-\delta)) \forall \delta>0 .
$$

Indeed, the sequence $\left\{\varphi_{n}\right\}$ can be assumed to converge to some $\varphi$ weakly in $L^{2}(\Omega \times$ 
$(0, T-\delta))$ for all $\delta>0$, since we have the estimates

$$
\begin{aligned}
\iint_{\Omega \times(0, T-\delta)}\left|\varphi_{n}\right|^{2} d x d t & \leq 2 \iint_{\Omega \times(0, T-\delta)}\left|p_{n}\right|^{2} d x d t+2 \iint_{\Omega \times(0, T-\delta)}\left|\zeta_{n}\right|^{2} d x d t \\
& \leq C_{\delta} \iint_{Q} e^{-2 C_{0} /(T-t)}\left|p_{n}\right|^{2} d x d t+C \\
& \leq C_{\delta} C_{1}\left\|\varphi_{n}^{T}\right\|_{h}^{2}+C \\
& =C_{\delta} C_{1}+C .
\end{aligned}
$$

Here, we have used (14). Obviously, $\varphi$ solves in $Q$ the PDE

$$
-\varphi_{t}-\Delta \varphi+\int_{\Omega} K(\xi, x) \varphi(\xi, t) d \xi=0
$$

and $\varphi(x, t)=0$ in $\omega \times(0, T)$; but this yields $\varphi \equiv 0$. Indeed, for $t$ a.e. in $(0, T)$, the function

$$
x \mapsto \int_{\Omega} K(\xi, x) \varphi(\xi, t) d \xi
$$

is analytic in $\Omega$ and vanishes in $\omega$, whence it vanishes everywhere; therefore, $\varphi$ is in fact a solution to the classical heat equation in $Q$ and, since it vanishes in $\omega \times(0, T)$, it is identically zero. This proves (22).

From (20), the fact that $\zeta_{n}$ is bounded in $L^{2}(Q)$ and (22), we deduce that

$$
\int_{\Omega} K(\xi, \cdot) \varphi_{n}(\xi, \cdot) d \xi \rightarrow 0 \text { weakly in } L^{2}\left(0, T ; H^{-1}(\Omega)\right)
$$

and, consequently, (19) holds.

Proof of (17). We will use the energy and observability estimates satisfied by $p$ and the energy estimates satisfied by $\zeta$.

More precisely, we see from (12) that

$$
\|p(\cdot, 0)\|^{2} \leq\|p(\cdot, t)\|^{2} \quad \forall t \in(0, T) .
$$

Using (14), we find that

$$
\|p(\cdot, 0)\|^{2} \leq C \int_{T / 4}^{3 T / 4}\|p(\cdot, t)\|^{2} d t \leq C\left\|\varphi^{T}\right\|_{h}^{2} .
$$

On the other hand, from (13) we have that

$$
\|\zeta(\cdot, 0)\|^{2} \leq C\left\|\int_{\Omega} K(\xi, \cdot) p(\xi, \cdot) d \xi\right\|_{L^{2}\left(0, T ; H^{-1}(\Omega)\right)}^{2}
$$

and, arguing as in (21), the following is found:

$$
\|\zeta(\cdot, 0)\|^{2} \leq C\|K\|_{R}^{2}\left\|\varphi^{T}\right\|_{h}^{2} .
$$

Finally, noting that $\varphi=p+\zeta$ and putting together (23) and (24), we get (17). This ends the proof of Theorem 3.

Copyright $@$ by SIAM. Unauthorized reproduction of this article is prohibited. 
Remark 6. Note that under the assumption (3), (1) is approximately controllable, that is, for any $y^{0}, y^{T} \in L^{2}(\Omega)$ and any $\epsilon>0$, there exist controls $v \in L^{2}(\omega \times(0, T))$ such that the corresponding solutions to (1) satisfy

$$
\left\|y(\cdot, T)-y^{T}\right\| \leq \epsilon .
$$

This is a consequence, via a well known argument, of the unique continuation property of the solutions to the adjoint systems (6). On the other hand, in view of the regularity of the solution to (1) outside $\omega \times(0, T)$, the exact controllability is obviously out of reach.

3. The nonlocal wave equation. Now, let us consider the hyperbolic system (2). Our second main result is the following.

Theorem 7. Assume that (3) holds and that the domain $\Omega$, the control subdomain $\omega$, and the time $T>0$ satisfy the classical GCC ensuring the observability and controllability properties of the linear wave equation:

$$
\left\{\begin{array}{l}
\partial \Omega \text { is of class } C^{3} \text { and } \\
(\omega, T) \text { satisfies the usual GCC in } \Omega .
\end{array}\right.
$$

Then, the linear system (2) is null-controllable.

Recall that, roughly speaking, $(\omega, T)$ satisfies the GCC in $\Omega$ if every ray of $g e$ ometric optics that starts at any point $x^{0} \in \Omega$ at time $t=0$ and travels with unit speed meets $\omega$ at a time $t<T$; see [2] and see also [3], where it is shown that the $C^{3}$ regularity of $\partial \Omega$ suffices to prove that GCC implies exact controllability. is

The proof of Theorem 7 is similar to that of Theorem 3. Now, the adjoint system

$$
\begin{cases}\psi_{t t}-\Delta \psi+\int_{\Omega} K(\xi, x) \psi(\xi, t) d \xi=0 & \text { in } Q \\ \psi=0 & \text { on } \Sigma \\ \psi(x, T)=\psi_{1}^{T}(x), \quad \psi_{t}(x, T)=\psi_{2}^{T}(x) & \text { in } \Omega\end{cases}
$$

and the observability estimate that we have to prove is

$$
\left\|\left(\psi, \psi_{t}\right)(\cdot, 0)\right\|_{L^{2} \times H^{-1}}^{2} \leq C \iint_{\omega \times(0, T)}|\psi|^{2} d x d t \quad \forall\left(\psi_{1}^{T}, \psi_{2}^{T}\right) \in L^{2}(\Omega) \times H^{-1}(\Omega) .
$$

Again, the main difficulty comes from the presence of the nonlocal term in the adjoint equation. However, in this case, this term is compact with respect to the natural $L^{2} \times H^{-1}$ norm of $\left(\psi_{1}^{T}, \psi_{2}^{T}\right)$ and this makes the argument easier to implement without requiring extra assumptions on the kernel $K$.

Assume that the hypotheses in Theorem 7 are satisfied. We will prove the estimate (27). write

For any $\left(\psi_{1}^{T}, \psi_{2}^{T}\right) \in L^{2}(\Omega) \times H^{-1}(\Omega)$, we denote by $\psi$ the solution to (26) and we

$$
\psi=q+\eta
$$

where $q$ is the solution to

$$
\begin{cases}q_{t t}-\Delta q=0 & \text { in } Q, \\ q=0 & \text { on } \Sigma \\ q(x, T)=\psi_{1}^{T}(x), \quad q_{t}(x, T)=\psi_{2}^{T}(x) & \text { in } \Omega\end{cases}
$$

Copyright (C) by SIAM. Unauthorized reproduction of this article is prohibited. 
and, consequently,

$$
\begin{cases}\eta_{t t}-\Delta \eta+\int_{\Omega} K(\xi, x) \eta(\xi, t) d \xi=-\int_{\Omega} K(\xi, x) q(\xi, t) d \xi & \text { in } Q \\ \eta=0 & \text { on } \Sigma \\ \eta(x, T)=0, \quad \eta_{t}(x, T)=0 & \text { in } \Omega .\end{cases}
$$

Thanks to (25), the mapping

$$
\left(\psi_{1}^{T}, \psi_{2}^{T}\right) \mapsto\left\|\left(\psi_{1}^{T}, \psi_{2}^{T}\right)\right\|_{w}:=\left(\iint_{\omega \times(0, T)}|q|^{2} d x d t\right)^{1 / 2}
$$

is a norm in $L^{2}(\Omega) \times H^{-1}(\Omega)$ and, moreover, there exists a constant $C_{2}(\Omega, \omega, T)$ such that

$$
\left\|\left(\psi_{1}^{T}, \psi_{2}^{T}\right)\right\|_{L^{2} \times H^{-1}}^{2} \leq C_{2}\left\|\left(\psi_{1}^{T}, \psi_{2}^{T}\right)\right\|_{w}^{2} \quad \forall\left(\psi_{1}^{T}, \psi_{2}^{T}\right) \in L^{2}(\Omega) \times H^{-1}(\Omega) .
$$

This is proved and explained in detail in [3].

As in the parabolic case, the inequality ( 7 ) will be a consequence of two estimates:

$$
\left\|\left(\psi_{1}^{T}, \psi_{2}^{T}\right)\right\|_{w}^{2} \leq C \iint_{\omega \times(0, T)}|\psi|^{2} d x d t \quad \forall\left(\psi_{1}^{T}, \psi_{2}^{T}\right) \in L^{2}(\Omega) \times H^{-1}(\Omega)
$$

and

$$
\left\|\left(\psi, \psi_{t}\right)(\cdot, 0)\right\|_{L^{2} \times H^{-1}}^{2} \leq C\left\|\left(\psi_{1}^{T}, \psi_{2}^{T}\right)\right\|_{w}^{2} \quad \forall\left(\psi_{1}^{T}, \psi_{2}^{T}\right) \in L^{2}(\Omega) \times H^{-1}(\Omega) .
$$

Proof of (30). Again, we argue by contradiction. Thus, let us assume that (30) does not hold and let the sequence $\left\{\left(\psi_{1, n}^{T}, \psi_{2, n}^{T}\right)\right\}$ satisfy

$$
1=\left\|\left(\psi_{1, n}^{T}, \psi_{2, n}^{T}\right)\right\|_{w}^{2}>n \iint_{\omega \times(0, T)}\left|\psi_{n}\right|^{2} d x d t \quad \forall n \in \mathbf{N},
$$

where $\psi_{n}$ is the solution to (26) corresponding to the final data $\left(\psi_{1, n}^{T}, \psi_{2, n}^{T}\right)$.

Let us denote by $q_{n}$ (resp. $\left.\eta_{n}\right)$ the solution to (28) associated to $\left(\psi_{1, n}^{T}, \psi_{2, n}^{T}\right)$ (resp. the solution to $(29)$ with $q$ replaced by $q_{n}$ ). Let us show that, at least for a subsequence, again denoted by $\left\{\psi_{n}\right\}$, one has

$$
\psi_{n} \rightarrow 0 \text { weakly-* in } L^{\infty}\left(0, T ; L^{2}(\Omega)\right)
$$

and

$$
\eta_{n} \rightarrow 0 \text { strongly in } L^{2}(Q) .
$$

The $\left(\psi_{1, n}^{T}, \psi_{2, n}^{T}\right)$ are uniformly bounded in $L^{2}(\Omega) \times H^{-1}(\Omega)$, whence

$$
\left\|\left(q_{n}, q_{n, t}\right)\right\|_{C^{0}\left([0, T] ; L^{2}(\Omega) \times H^{-1}(\Omega)\right)} \leq C \quad \forall n \in \mathbf{N} .
$$

Therefore, the right-hand sides of the PDEs satisfied by the $\eta_{n}$ are uniformly bounded in $C^{0}\left([0, T] ; L^{2}(\Omega)\right)$ and

$$
\left\|\left(\eta_{n}, \eta_{n, t}\right)\right\|_{C^{0}\left([0, T] ; H_{0}^{1}(\Omega) \times L^{2}(\Omega)\right)} \leq C \quad \forall n \in \mathbf{N} .
$$

Copyright $@$ by SIAM. Unauthorized reproduction of this article is prohibited. 
From (35) and (36), we see that $\psi_{n}$ is uniformly bounded in $C^{0}\left([0, T] ; L^{2}(\Omega)\right)$ and we can assume that $\psi_{n}$ converges weakly-* in $L^{\infty}\left(0, T ; L^{2}(\Omega)\right)$ to a function $\psi$. Clearly, $\psi$ solves in $Q$ the PDE

$$
\psi_{t t}-\Delta \psi+\int_{\Omega} K(\xi, x) \psi(\xi, t) d \xi=0
$$

and $\psi(x, t)=0$ in $\omega \times(0, T)$. But this implies $\psi \equiv 0$, in view of the analyticity of the function

$$
x \mapsto \int_{\Omega} K(\xi, x) \psi(\xi, t) d \xi
$$

and the GCC satisfied by $(\omega, T)$. Hence, (33) holds.

In order to prove (34), it suffices to check that $\eta_{n}$ belongs to a compact set of $L^{2}(Q)$. But this is evident, in view of (36) and a standard application of the Aubin-Lions Lemma.

We find a contradiction: (32) and (34) are not compatible with the fact that $\left\|\left(\psi_{1, n}^{T}, \psi_{2, n}^{T}\right)\right\|_{w}=1$ for all $n$, since

$$
\left\|\left(\psi_{1, n}^{T}, \psi_{2, n}^{T}\right)\right\|_{w}^{2}=\iint_{\omega \times(0, T)}\left|q_{n}\right|^{2} d x d t \leq 2 \iint_{\omega \times(0, T)}\left|\psi_{n}\right|^{2} d x d t+2 \iint_{\omega \times(0, T)}\left|\eta_{n}\right|^{2} d x d t .
$$

Proof of (31). This is easy since, for any $\left(\psi_{1}^{T}, \psi_{2}^{T}\right) \in L^{2}(\Omega) \times H^{-1}(\Omega)$, one has

$$
\begin{aligned}
\left\|\left(\psi, \psi_{t}\right)(\cdot, 0)\right\|_{L^{2} \times H^{-1}}^{2} & \leq 2\left\|\left(q, q_{t}\right)(\cdot, 0)\right\|_{L^{2} \times H^{-1}}^{2}+2\left\|\left(\eta, \eta_{t}\right)(\cdot, 0)\right\|_{L^{2} \times H^{-1}}^{2} \\
& \leq 2\left\|\left(\psi_{1}^{T}, \psi_{2}^{T}\right)\right\|_{L^{2} \times H^{-1}}^{2}+C \iint_{Q}|q|^{2} d x d t \\
& \leq C \iint_{\omega \times(0, T)}|q|^{2} d x d t \\
& =C\left\|\left(\psi, \psi_{t}\right)(\cdot, 0)\right\|_{w}^{2} .
\end{aligned}
$$

From (30) and (31), we deduce (27). This ends the proof of Theorem 7.

4. Some additional comments and questions. In this paper, we have only considered some very simple models. There are many interesting and challenging related problems for equations with nonlocal terms to be investigated. Some of them are mentioned below.

- Can the analyticity assumption on $K$ be removed?

Note that the analyticity assumption is doubly needed for the heat equation in order to implement the compactness-uniqueness argument. But, at the level of unique continuation, whether this analyticity assumption is needed for the heat and wave equations (6) and (26) is an interesting open problem.

- Can one employ Carleman inequalities to prove the observability estimate of equations with nonlocal terms?

To our best knowledge, until now, there has been no direct proof of the observability estimate for equations with nonlocal terms achieved by means of Carleman-type inequality. The main difficulty, as we said in Remark 4, is that, in (9), terms like

$$
\varepsilon \iint_{\Omega \times(0, T)} \rho^{-2}\left|\int_{\Omega} K(\xi, x) \varphi(\xi, t)\right|^{2} d x d t
$$

Copyright $@$ by SIAM. Unauthorized reproduction of this article is prohibited. 
cannot be absorbed by the terms in the left-hand side. It would be interesting to analyze whether one can choose suitable weight functions to establish Carleman inequalities adapted to deal with nonlocal terms.

- Boundary control systems.

Whether the results of this paper can be extended to boundary control problems is also an interesting open problem.

For both the wave and the heat equation involving nonlocal terms it is unclear whether the compactness-uniqueness argument can be applied. Note that the unique continuation argument we have employed, based on the analyticity of the kernel with respect to $x$, does not hold.

In particular, is it true that for the solutions of the nonlocal adjoint heat equation (6) the fact that the normal derivative of the solution vanishes on the boundary implies that the solution vanishes everywhere? The same question can be formulated for the wave equation.

- Systems with nonlocal terms of higher order.

We can also consider systems of the form

$$
\begin{cases}y_{t}-\int_{\Omega} K(x, \xi) \Delta y(\xi, t) d \xi=v 1_{\omega} & \text { in } Q \\ y=0 & \text { on } \Sigma \\ y(x, 0)=y^{0}(x) & \text { in } \Omega\end{cases}
$$

involving nonlocal terms in the principal part of the heat operator, under adequate hypotheses on $K$. This is a model with interesting physical applications (see [1] for an introduction to this topic). But, obviously, the compactnessuniqueness argument employed in this paper cannot be directly applied or adapted to establish the null controllability of (37).

It would be interesting to explore the class of nonlocal coefficients for which null controllability holds for this model.

- Nonlinear control problems.

The results in this paper could also be seen as a first step to handle models involving nonlocal nonlinear terms. But significant further developments are needed in this direction.

\section{REFERENCES}

[1] F. Andréu-Vaillo, J. M. Mazón, J. D. Rossi, and J. J. Toledo-Melero, Nonlocal Diffusion Problems, Math. Surveys Monogr. 165, AMS, Providence, RI, 2010.

[2] C. Bardos, G. Lebeau, and J. Rauch, Sharp sufficient conditions for the observation, control and stabilization of waves from the boundary, SIAM J. Control Optim., 30 (1992), pp. 10241065.

[3] N. BurQ, Contrôlabilité exacte des ondes dans des ouverts peu réguliers, Asymptot. Anal., 14 (1997), pp. 157-191.

[4] M. De Leo, C. S. F. De la Vega, And D. Rial, Controllability of Schrödinger equation with a nonlocal term, ESAIM Control Optim. Calc. Var., 20 (2014), pp. 23-41.

[5] E. FernándeZ-Cara And E. Zuazua, The cost of approximate controllability for heat equations: The linear case, Adv. Differential Equations, 5 (2000), pp. 465-514.

[6] E. Fernández-CARA ANd E. ZuAZuA, Null and approximate controllability for weakly blowing up semilinear heat equations, Ann. Inst. H. Poincaré Anal. Non Linéaire, 17 (2000), pp. 583616.

[7] A. V. Fursikov and O. Yu. Imanuvilov, Controllability of Evolution Equations, Lecture Notes Ser. 34, Seoul National University, Research Institute of Mathematics, Global Analysis Research Center, Seoul, 1996.

[8] P. I. Naumkin And I. A. Shishmarëv, Nonlinear Nonlocal Equations in the Theory of Waves, Transl. Math. Monogr. 133, AMS, Providence, RI, 1994.

[9] A. Okubo And S. A. Levin, Diffusion and Ecological Problems: Modern Perspectives, 2nd ed., Interdiscip. Appl. Math. 14. Springer-Verlag, New York, 2001. 